\title{
Predicting aggressive behavior in small renal tumors prior to treatment
}

\author{
Daniel D. Shapiro, E. Jason Abel \\ Department of Urology, University of Wisconsin School of Medicine and Public Health, Madison, WI, USA \\ Correspondence to: E. Jason Abel, MD, FACS. Department of Urology, University of Wisconsin School of Medicine and Public Health, 1685 Highland \\ Avenue, Madison, WI 53705, USA. Email: abel@urology.wisc.edu. \\ Provenance: This is an invited Editorial commissioned by Section Editor Xiao Li (Department of Urology, Jiangsu Cancer Hospital \& Jiangsu \\ Institute of Cancer Research \& Nanjing Medical University Affiliated Cancer Hospital, Nanjing, China). \\ Comment on: Bhindi B, Thompson RH, Lohse CM, et al. The Probability of Aggressive Versus Indolent Histology Based on Renal Tumor Size: \\ Implications for Surveillance and Treatment. Eur Urol 2018;74:489-97.
}

Submitted Nov 19, 2018. Accepted for publication Dec 17, 2018.

doi: $10.21037 /$ atm.2018.12.46

View this article at: http://dx.doi.org/10.21037/atm.2018.12.46

With widespread utilization of cross sectional imaging, the incidence of renal tumors continues to increase and tumors $\leq 4 \mathrm{~cm}$ comprise $48-66 \%$ of new renal cell carcinoma (RCC) diagnoses (1). The increased detection of small incidental tumors and technological improvements in surgery may lead to overtreatment of co-morbid patients with slow growing tumors (2). In order to risk stratify patients, a recent study by Bhindi et al. has proposed dividing small renal mass (SRM) patients into either indolent or aggressive pathologic subgroups based on associations of radiographic size and gender with aggressive tumor histology (3). Improved understanding of the individual risks associated with aggressive SRM may facilitate counseling patients considering treatment. However, accurately determining individual SRM behavior remains difficult, and treatment decisions may not be straightforward.

In general, SRMs fall into three general categories: benign tumors, aggressive cancers, and indolent cancers (Figure 1). After radiographic diagnosis, physicians must weigh the patient's cancer risk in the context of his or her overall health to determine the benefit of SRM treatment. Surgery is considered a standard treatment for SRMs, which has the advantage of removing the entire primary tumor to allow for the most complete histologic evaluation. However, treatment of SRMs using thermal ablation or active surveillance (AS) (i.e., deferring treatment while evaluating growth on serial imaging) have emerged as recommended options, especially for older patients with co-morbid illnesses or limited life expectancy $(6,7)$. As the options for

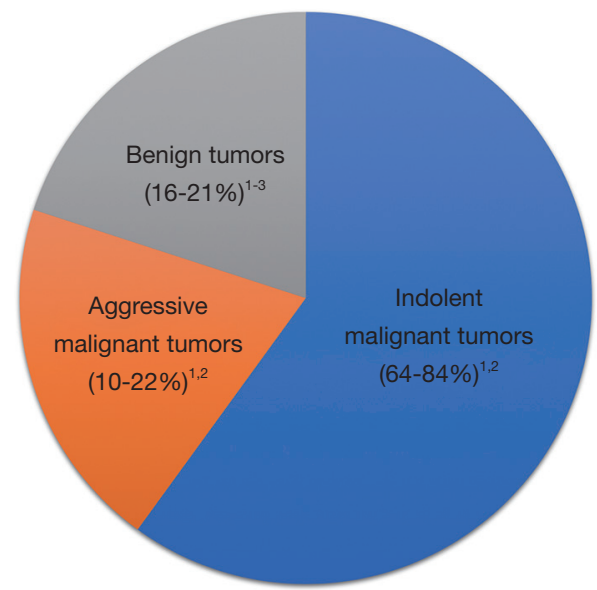

Figure 1 Etiologies of renal tumors $\leq 4 \mathrm{~cm} .{ }^{1}$, Bhindi et al. (3); ${ }^{2}$, Thompson et al. (4); ${ }^{3}$, Kutikov et al. (5).

SRM treatment continue to evolve, patients and physicians have an opportunity to improve shared decision making and individual outcomes by understanding current prognostic data and by developing new prognostic biomarkers.

The median age of diagnosis for cancer of the kidney and renal pelvis is 64 years old (8). After treatment, tenyear cancer mortality from non-metastatic small RCC is low (Figure 2), with a recent large single institutional series demonstrating approximately $7 \%$ cancer mortality at 10 years for tumors $\leq 4 \mathrm{~cm}$ (3). Ten-year cancer mortality was similar in a prior multi-center study at $8.6 \%$ (9). These 


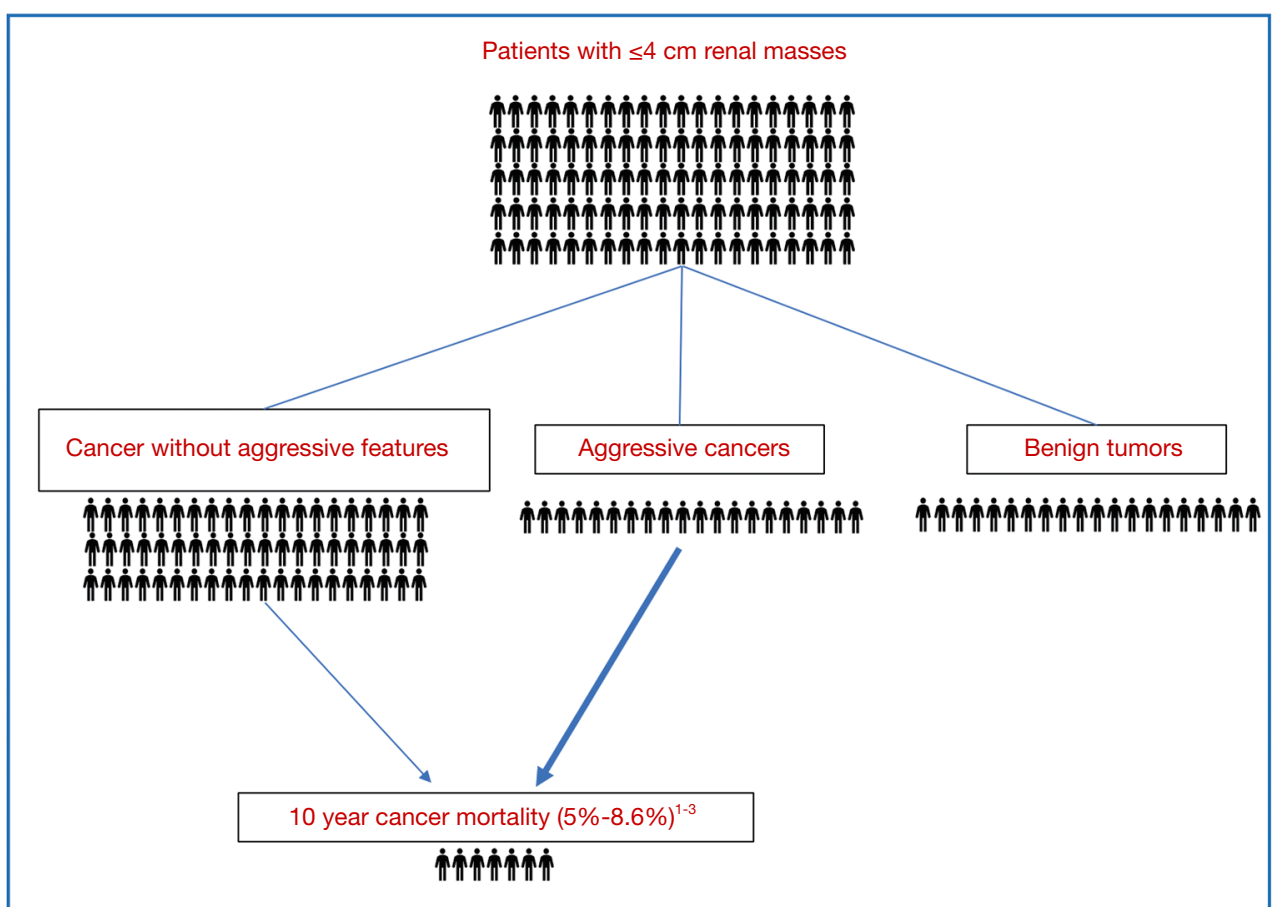

Figure 2 Cancer specific outcomes for patients treated surgically for renal masses $\leq 4 \mathrm{~cm} .{ }^{1}$, Bhindi et al. (3); ${ }^{2}$, Ficarra et al. (9); ${ }^{3}$, Kutikov et al. (10).

findings are also comparable to a study of 30,801 localized RCC patients from the Surveillance, Epidemiology, and End Results (SEER) database that estimated a 5\% risk of cancer death at 10 years following surgery for patients with tumors $<4 \mathrm{~cm}$ (10). The authors also found RCC 10-year mortality was significantly lower compared to 10 -year probability of non-cancer death (14-66\%) or death from a different cancer $(5-11 \%)$ for patients $\geq 50$ years old (10). Observations that small RCC have a low overall risk of cancer mortality and a slow time to metastatic progression provided evidence for development of AS protocols particularly for older patients with significant comorbidities (11). Newer prospective studies have shown that AS could be an initial option for many patients and surveillance was recently discussed in RCC treatment guidelines (6).

Given the low risk of cancer mortality for small RCC $(3,10)$, clinically meaningful prognostic markers must be associated with a substantial increase or decrease in risk. For example, identification of benign tumors [e.g., oncocytoma or angiomyolipoma (AML)] greatly decreases the risk of cancer mortality and therefore this prognostic information may significantly impact treatment decision making. Percutaneous biopsy reliably identifies benign versus malignant renal masses, and for the $20 \%$ of people found to have a benign SRMs after biopsy (Figure 2), the risk of cancer mortality decreases dramatically compared to those with RCC (12). There remains a concern that some small malignant eosinophilic tumors are misdiagnosed as oncocytomas because of limited tissue sampling with biopsy (13), and surgery remains the preferred longterm management for larger oncocytic tumors in younger patients. However, many patients with small oncocytic neoplasms are ideal candidates for AS $(14,15)$.

Since many SRMs are incidental and diagnosed from imaging, radiographic size is a simple available metric to use for prognosis. Bhindi et al. demonstrated that radiographic size is prognostic for aggressive tumor histology in pT1 or pT2 patients, which expands on previous work demonstrating that tumor size predicts benign versus malignant histology (3). For $2 \mathrm{~cm}$ tumors, there was a lower risk of aggressive pathology when compared to $4 \mathrm{~cm}$ tumors ( $18 \%$ vs. $29 \%)$, but the risk was highest $(53 \%)$ for tumors $>10 \mathrm{~cm}$. As described previously (16), female RCC patients have slightly improved outcomes with lower risk of aggressive pathology compared to male patients (3). Another interesting conclusion that can be drawn from this study is that even high-quality conventional pathology is inadequate to identify patients at risk of RCC progression. After 
experienced genitourinary pathologist review at a center of excellence, $42 \%$ of patients with metastatic progression and $33 \%$ of those who died from RCC had primary tumors that were classified as indolent cancers (3). The inability of conventional pathology to identify aggressive histology for 1 in 3 patients dying from RCC might be from sampling error within heterogeneous tumors (17), the inability to measure differences in host immunologic factors, or an inherent shortcoming of conventional histology techniques that may improve with molecular RCC subtyping (18).

Tumor volume is a prognostic marker that may be superior to tumor diameter, with greater concordance between radiographic volumes, pathologic volumes, and tumor grade (19). Tumor growth (change in tumor size or volume over time) has prognostic value for patients on AS, with faster growth rates associated with the risk of tumor progression. In prospective studies, SRMs often demonstrate indolent growth (20), with higher one-dimensional growth rates $(0.8 v s .0 .3 \mathrm{~cm} /$ year $)$ and volumetric growth rate $\left(27.1 \mathrm{vs} .6 .2 \mathrm{~cm}^{3} /\right.$ year) associated with aggressive tumors (21). With AS becoming more widely utilized $(20,21)$, tumor growth will likely continue to be an important prognostic marker to identify tumors that may benefit from active treatments.

\section{Advanced imaging techniques}

Conventional imaging is dependent on expert interpretation, which may vary significantly between radiologists or among imaging modalities. Texture analysis is an active area of research which utilizes data from computed tomographic (CT) or magnetic resonance imaging (MRI) and postprocessing analyses to evaluate imaging features such as spatial heterogeneity, which could be biomarkers for tumor behavior. Texture features have been correlated with adverse tumor biology including angiogenesis, cell death, and cellular proliferation. In RCC, CT texture analysis has shown to be prognostic for clear cell and non-clear cell histologic subtypes as well as tumor grade and tissue expression of Ki-67, C reactive protein and neovascularity (22). Other promising imaging modalities for advanced RCC use positron emission tomography/CT to investigate tumor angiogenesis (23) or target cell surface proteins such as carbonic anhydrase IX, which is enriched in RCC tumors (24). Although advanced imaging techniques have primarily been investigated in advanced or metastatic RCC, the future role of imaging biomarkers for SRM evaluation may expand if accurate, non-invasive methods are developed to identify aggressive tumors.

\section{Advanced molecular analysis}

Developing molecular RCC prognostic biomarkers is an active area of investigation, which may involve one of several advanced techniques to analyze tumor tissue or patient serum. Similar to advanced imaging in RCC, molecular biomarkers have largely been investigated in patients with advanced or metastatic tumors. Molecular characterization of RCC has moved beyond traditional pathologic grouping of tumors into histologic subtypes, suggesting that many subgroups and individual prognostic signatures can be identified within each group. The Cancer Genome Atlas (TCGA) network evaluated 843 RCC specimens to determine survival differences based on histology as well as molecular characterization including hypermethylation, mRNA signatures, metabolic gene expression, and immune signature analysis. Increased ribose metabolism mRNA are associated with decreased survival in clear cell carcinoma (25). This study further demonstrated DNA hypermethylation/CDKN2A alterations associate with worse survival in all RCC subtypes (25). Refining these molecular subtypes may help to provide a more individualized prognosis and find further targets for therapy. For example, the loss of $C D K N 2 A$ correlates with worse prognosis in clear cell, papillary, and chromophobe RCC and may be targetable with CDK4/6 inhibitors (25). Type 2 papillary RCC has increased ribose metabolism suggesting a more metabolically active tumor, likely explaining its worse survival compared to type 1 papillary RCC, which may be a pathway to target for future therapy (25). Other studies have looked at potential biomarkers to determine localized RCC tumor prognosis. Qu et al. evaluated the prognostic value of long non-coding RNA (lncRNA) to determine localized RCC prognosis (26). Through interactions with protein kinases, cell receptors and transcription factors, lncRNAs regulate enzymatic activities within cancer cells. Using TCGA data, a four-lncRNA-based classifier was constructed, termed "RCClnc4 signature". Increased expression of the four selected lncRNAs was an independent predictor of overall survival as well as disease specific survival. The RCClnc4 signature was more accurate compared to clinical staging systems such as TNM and the stage, size, grade and necrosis (SSIGN) score (26), although the overall predictive accuracy of RCClnc4 signature was only modest (mean C-index 0.72). Future studies should continue to refine molecular diagnostic tests and improve their predictive 
accuracy, so that utilization of advanced techniques will enable personalized treatment strategies for localized RCC.

One ubiquitous criticism of tissue-based biomarkers for RCC is the inevitable risk of sampling error given the known intra-tumor heterogeneity in large tumors (17). Recent studies suggest that defining "evolutionary subtypes" of mutational analyses may be prognostic for progression in RCC, providing a possible framework to interpret tissue heterogeneity in RCC (27). To avoid this confusion with tissue sampling errors, a serum test to provide RCC prognostic information seems ideal. Multiple platforms have been developed to test circulating DNA in patients with RCC, most of which are currently being investigated in metastatic RCC (28). Currently, it is unclear how circulating DNA or other emerging serum tests will perform for patients with small less aggressive localized tumors, although the advantages of identifying an accurate serum RCC marker are self-evident.

In conclusion, small renal tumors can be generalized into three categories: benign tumors, aggressive cancers, and indolent cancers with multiple studies demonstrating excellent long-term cancer outcomes following treatment. Currently, the most impactful and reliable prognostic information is obtained by identifying common benign tumors (e.g., oncocytoma and typical AML) with biopsy. For patients with small RCC, tumor diameter, gender and conventional pathologic features are prognostic for progression. Decision making for treatment of small renal tumors is based on clinical and pathologic features; however, a significant proportion of patients with small RCC who progress to metastatic cancer are not identified by current pathologic techniques. Future advances in imaging and molecular analysis will likely improve prognostic ability for RCC risk stratification and facilitate an individualized approach to decision making.

\section{Acknowledgements}

None.

\section{Footnote}

Conflicts of Interest: The authors have no conflicts of interest to declare.

\section{References}

1. Nguyen MM, Gill IS, Ellison LM. The evolving presentation of renal carcinoma in the United States: trends from the Surveillance, Epidemiology, and End Results program. J Urol 2006;176:2397-400; discussion 2400.

2. Shah PH, Alom MA, Leibovich BC, et al. The Temporal Association of Robotic Surgical Diffusion with Overtreatment of the Small Renal Mass. J Urol 2018;200:981-8.

3. Bhindi B, Thompson RH, Lohse CM, et al. The Probability of Aggressive Versus Indolent Histology Based on Renal Tumor Size: Implications for Surveillance and Treatment. Eur Urol 2018;74:489-97.

4. Thompson RH, Atwell T, Schmit G, et al. Comparison of partial nephrectomy and percutaneous ablation for cT1 renal masses. Eur Urol 2015;67:252-9.

5. Kutikov A, Fossett LK, Ramchandani P, et al. Incidence of benign pathologic findings at partial nephrectomy for solitary renal mass presumed to be renal cell carcinoma on preoperative imaging. Urology 2006;68:737-40.

6. Campbell S, Uzzo RG, Allaf ME, et al. Renal Mass and Localized Renal Cancer: AUA Guideline. J Urol 2017;198:520-9.

7. Finelli A, Ismaila N, Bro B, et al. Management of Small Renal Masses: American Society of Clinical Oncology Clinical Practice Guideline. J Clin Oncol 2017;35:668-80.

8. Noone A, Howlader N, Krapcho M, et al. SEER Cancer Statistics Review, 1975-2015, National Cancer Institute. Bethesda, MD, based on November 2017 SEER data submission, posted to the SEER web site, April 2018. Available online: https://seer.cancer.gov/csr/1975_2015/

9. Ficarra V, Schips L, Guille F, et al. Multiinstitutional European validation of the 2002 TNM staging system in conventional and papillary localized renal cell carcinoma. Cancer 2005;104:968-74.

10. Kutikov A, Egleston BL, Wong YN, et al. Evaluating Overall Survival and Competing Risks of Death in Patients With Localized Renal Cell Carcinoma Using a Comprehensive Nomogram. J Clin Oncol 2010;28:311-7.

11. Jewett MA, Mattar K, Basiuk J, et al. Active surveillance of small renal masses: progression patterns of early stage kidney cancer. Eur Urol 2011;60:39-44.

12. Marconi L, Dabestani S, Lam TB, et al. Systematic Review and Meta-analysis of Diagnostic Accuracy of Percutaneous Renal Tumour Biopsy. Eur Urol 2016;69:660-73.

13. Patel HD, Druskin SC, Rowe SP, et al. Surgical histopathology for suspected oncocytoma on renal mass biopsy: a systematic review and meta-analysis. BJU Int 2017;119:661-6.

14. Richard PO, Jewett MA, Bhatt JR, et al. Active 
Surveillance for Renal Neoplasms with Oncocytic Features is Safe. J Urol 2016;195:581-6.

15. Miller BL, Mankowski Gettle L, Van Roo JR, et al. Comparative Analysis of Surgery, Thermal Ablation, and Active Surveillance for Renal Oncocytic Neoplasms. Urology 2018;112:92-7.

16. Abel EJ, Culp SH, Meissner M, et al. Identifying the risk of disease progression after surgery for localized renal cell carcinoma. BJU Int 2010;106:1277-83.

17. Gerlinger M, Rowan AJ, Horswell S, et al. Intratumor heterogeneity and branched evolution revealed by multiregion sequencing. N Engl J Med 2012;366:883-92 .

18. Brugarolas J. Molecular genetics of clear-cell renal cell carcinoma. J Clin Oncol 2014;32:1968-76.

19. Choi SM, Choi DK, Kim TH, et al. A comparison of radiologic tumor volume and pathologic tumor volume in renal cell carcinoma (RCC). PLoS One 2015;10:e0122019.

20. Pierorazio PM, Johnson MH, Ball MW, et al. Five-year analysis of a multi-institutional prospective clinical trial of delayed intervention and surveillance for small renal masses: the DISSRM registry. Eur Urol 2015;68:408-15.

21. Smaldone MC, Kutikov A, Egleston BL, et al. Small renal masses progressing to metastases under active surveillance: a systematic review and pooled analysis. Cancer 2012;118:997-1006.

Cite this article as: Shapiro DD, Abel EJ. Predicting aggressive behavior in small renal tumors prior to treatment. Ann Transl Med 2018;6(Suppl 2):S132. doi: 10.21037/atm.2018.12.46
22. Scrima AT, Lubner MG, Abel EJ, et al. Texture analysis of small renal cell carcinomas at MDCT for predicting relevant histologic and protein biomarkers. Abdom Radiol (NY) 2018. [Epub ahead of print].

23. Evangelista L, Basso U, Maruzzo M, et al. The Role of Radiolabeled Prostate-specific Membrane Antigen Positron Emission Tomography/Computed Tomography for the Evaluation of Renal Cancer. Eur Urol Focus 2018. [Epub ahead of print].

24. Minn I, Koo SM, Lee HS, et al. [64Cu]XYIMSR-06: A dual-motif CAIX ligand for PET imaging of clear cell renal cell carcinoma. Oncotarget 2016;7:56471-9.

25. Ricketts CJ, De Cubas AA, Fan H, et al. The Cancer Genome Atlas Comprehensive Molecular Characterization of Renal Cell Carcinoma. Cell Rep 2018;23:313-26.e5.

26. Qu L, Wang ZL, Chen Q, et al. Prognostic Value of a Long Non-coding RNA Signature in Localized Clear Cell Renal Cell Carcinoma. Eur Urol 2018;74:756-63.

27. Mitchell TJ, Turajlic S, Rowan A, et al. Timing the Landmark Events in the Evolution of Clear Cell Renal Cell Cancer: TRACERx Renal. Cell 2018;173:611-23.e17.

28. Bergerot PG, Hahn AW, Bergerot CD, et al. The Role of Circulating Tumor DNA in Renal Cell Carcinoma. Curr Treat Options Oncol 2018;19:10. 